\section{Surface hydrophobicity of the rat colonic mucosa is a defensive barrier against macromolecules and toxins}

A Lugea, A Salas, J Casalot, F Guarner, J-R Malagelada

\begin{abstract}
Background-Mucosal surface hydrophobicity is a key factor of the gastric acid defence barrier. In the colon, surface hydrophobicity is high but its biological function remains unexplored.
\end{abstract}

Aims-To investigate the functional changes of the barrier due to removal of the surface active phospholipid layer by a detergent, or to reinforcement of the surface active phospholipid by local application of a suspension of lipids.

Methods-Surface hydrophobicity (contact angle measurement), colonic permeability (lumen to blood clearance of mannitol and dextran), and mucosal resistance against luminal aggression (distal colitis induced by dextran sodium sulphate, DSS) were investigated in three study groups: $(a)$ rats pretreated with a detergent (Brij 35) known to remove surfactant lipids; $(b)$ rats pretreated with a suspension of surface active lipids (tripalmitin and dipalmitoyl-phosphatidylcholine); and (c) control rats pretreated with the corresponding vehicles. Results-In controls, surface hydrophobicity was low on the caecal mucosa and high in colon and rectum. Detergent treatment reduced surface hydrophobicity, and increased colonic permeability to mannitol and dextran. Conversely, treatment with lipids increased surface hydrophobicity, and reduced colonic permeability. Administration of DSS induced a progressive loss of colonic surface hydrophobicity, and an increase in permeability to mannitol and dextran. Detergent treatment increased susceptibility to epithelial damage and mucosal inflammation by DSS. Treatment with lipids reduced susceptibility to DSS colitis.

Conclusion-Colonic surface hydrophobicity modulates permeability to hydrophilic molecules and protects against toxins.

(Gut 2000;46:515-521)

Keywords: mucosal surface hydrophobicity; mannitol; dextran

The hydrophobic character of gastrointestinal mucus seems to be a critical factor in the defence of the gastroduodenal mucosa against luminal acid. $^{1-3}$ Surface hydrophobicity is attributable to a layer of surface active lipids which lines the top of the mucus that covers the epithelium. ${ }^{4-6}$ The surface active phospholipid layer protects against luminal acidity by repelling the diffusion of hydrogen ions. ${ }^{7}$ In several mammalian species including humans, surface hydrophobicity is very high on top of the gastric mucosa, whereas it is much lower throughout the small intestine which is the absorptive surface. Surface hydrophobicity in the colon is as high as in the upper tract, ${ }^{8-10}$ but its biological function remains mostly unexplored.

A large variety of bacterial populations with different pathogenic potentials and a high concentration of chemical and bacterial toxins are present within the colonic lumen. These are constantly challenging the structure and function of the mucosa. As shown for the gastric mucosa, ${ }^{1}$ surface hydrophobicity in the colon may prevent the influx of water soluble bacterial products and toxins, and could thereby provide an effective mechanism of protection for surface epithelial cells. Hence, our aim was to explore the role of surface hydrophobicity on colonic mucosal barrier function. We conducted a series of studies in the rat, looking at the functional changes of the barrier due either to removal of the surface active phospholipid layer by a detergent, or to reinforcement of the surface active phospholipid by local application of a suspension of lipids.

\section{Materials and methods}

ANIMALS AND PROTOCOLS

All experiments were performed on pathogen free male Sprague-Dawley rats weighing 200$250 \mathrm{~g}$, purchased from Centre d'Elevage R Janvier (Le Genest, France). The rats were housed under controlled conditions of temperature, humidity, and illumination, and maintained on standard rodent chow (Letica, Barcelona, Spain) and tap water. All studies were approved by the local research committee (Comissio de Recerca, Hospital General Vall d'Hebron).

Three protocols were included in this study. The first protocol studied mucosal surface hydrophobicity of the colon. The second protocol investigated colonic permeability by measuring the lumen to blood clearance of labelled molecules. The third protocol investigated the resistance of the colonic mucosa against luminal aggression. In each protocol, the effects of either removing the colonic

Abbreviations used in this paper: DSS, dextran sodium sulphate; TNBS, trinitrobenzenesulphonic acid; TP-PC, tripalmitin and dipalmitoyl-phosphatidylcholine. 
surface active phospholipid layer by a detergent, or strengthening the phospholipid layer by topical application of a lipid suspension were studied. Thus, all experiments included three groups of rats. The first group consisted of control rats that received no specific treatment, but only the vehicle used in the matched test group. A second group consisted of rats treated with the non-ionic detergent Brij 35 (5\% polyoxyethylene 23 lauryl ether, Sigma, St Louis, Missouri), that was shown to remove surface active phospholipid from the surface of the proximal duodenum. ${ }^{3}$ The detergent was given either by enema through a soft cannula ( $2 \mathrm{ml}$ of a $5 \%$ solution of Brij 35 in distilled water) or orally in the drinking water as a $2 \%$ solution of Brij 35 in tap water for two days. Finally, a third group of rats received intracolonically a $2 \mathrm{ml}$ bolus of tripalmitin (Sigma) and dipalmitoyl-phosphatidylcholine (Sigma) in $0.9 \%$ saline at a $2: 1$ ratio (TP-PC, $1.5 \mathrm{mg}$ lipid per $\mathrm{ml}$ ). The suspension was prepared as described by Lichtenberger et al. ${ }^{11}$

\section{MUCOSAL SURFACE HYDROPHOBICITY}

The hydrophobic surface properties of the colonic mucosa were studied in rats subjected to the above mentioned treatments. Rats were killed by cervical dislocation, the colon was removed, opened along the antimesenteric line, and rinsed with $0.9 \%$ saline at room temperature. The colon was divided in five sections: rectum (up to $2.5 \mathrm{~cm}$ from anus), distal colon (from 3 to $8 \mathrm{~cm}$ ), midcolon (from 8 to $13 \mathrm{~cm}$ ), proximal colon (from 13 to $18 \mathrm{~cm}$ ), and caecum, which is macroscopically distinct in the rat. Specimens were placed on a glass microscope slide, mucosal side up, and prepared for measurement of surface hydrophobicity by determination of the contact angle that conforms a microdrop of water deposited on the mucosal surface, as described previously. ${ }^{12}{ }^{13}$ The contact angle is the angle between the solid surface and the tangent to the liquid-air interface at the triple point where all three phases meet (solid, liquid, air). The surface was allowed to dry for 30 minutes at room temperature, and the specimen was then transferred to the stage of a goniometer (model 100-00, Ramé-Hart, Mountain Lakes, New Jersey). A $5 \mu \mathrm{l}$ droplet of saline was carefully laid on top of the mucosa, and the contact angle formed at the interface was measured using the inbuilt rules and scale of the goniometer. Large contact angles are read on hydrophobic surfaces whereas small angles are formed on hydrophillic surfaces.

COLONIC PERMEABILITY

To assess permeability, lumen to blood clearance of ${ }^{14} \mathrm{C}-\mathrm{D}$-mannitol (molecular weight 184 daltons; Amersham International, Buckinghamshire, UK) and ${ }^{3} \mathrm{H}$-dextran (molecular weight 70000 daltons; Amersham) was measured in rats anaesthetised with $1.25 \mathrm{~g} / \mathrm{kg}$ intraperitonal urethane (Fluka Chemie, Buchs, Switzerland). A tracheostomy was performed and the right carotid artery and left jugular vein were cannulated with polyethylene tubing (PE50, Clay Adams, Becton Dickinson, Parsip- pany, New Jersey, USA). A midline abdominal incision was performed, the colon was exteriorised, and bilateral nephrectomy was achieved by ligation of the renal pedicles. The colon was cannulated at both the hepatic flexure and the rectum using polyethylene tubing (internal diameter 1 and $7 \mathrm{~mm}$, respectively). The luminal contents were removed by injection of $20 \mathrm{ml}$ Krebs-Ringer-Tris solution ( $\mathrm{pH}$ 7.4) and $10 \mathrm{ml}$ air through the proximal cannula before the insertion of the rectal cannula through the anal canal. The colon was then replaced in the abdominal cavity and the incision was closed by suture.

For each study, a test solution was prepared consisting of $2.4 \mathrm{ml}$ of $10 \mathrm{mM}$ cold mannitol in Krebs-Ringer-Tris ( $\mathrm{pH}$ 7.4) with $0.6 \mu \mathrm{Ci}$ ${ }^{14} \mathrm{C}$-mannitol and $0.3 \mu \mathrm{Ci}{ }^{3} \mathrm{H}$-dextran. A $2 \mathrm{ml}$ bolus of the solution was instilled through the proximal cannula into the colonic lumen as a bolus and both cannulas were kept closed for five hours. Total radioactivity administered to each animal was calculated by counting two $200 \mu \mathrm{l}$ aliquots of the solution using a scintillation counter with a dual channel programme for simultaneous ${ }^{14} \mathrm{C}$ and ${ }^{3} \mathrm{H}$ counting (LS 6500, Beckman Instruments, Palo Alto, California). Blood samples $(500 \mu \mathrm{l})$ were taken through the carotid line at zero, one, two, three, four, and five hours after the bolus, and used for measurement of plasma radioactivity by means of the scintillation counter. Immediately after sampling through the carotid line, an equal volume of $2 \%$ albumin in saline was administered via the jugular catheter to prevent changes in haemodynamics. Finally, the rat was killed by anaesthetic overdose, and two urine samples were obtained from the bladder and counted to exclude leakage of the radioactive probes. Data on lumen to blood clearance of mannitol are expressed as percentage of the administered dose that accumulated in the extracellular fluid volume $(25 \%$ of body weight) during the five hours follow up. Lumen to blood clearance of dextran is expressed as percentage of the administered dose that accumulated in the intravascular fluid volume $(6 \%$ of body weight).

\section{MUCOSAL RESISTANCE AGAINST LUMINAL}

AGGRESSION

The role of the colonic surface active phospholipid as a protective barrier of the mucosa against luminal aggression was studied using a model of distal colitis induced by oral dextran sodium sulphate (DSS). The exact mechanism of DSS induced mucosal injury is not fully understood, but a topical toxic effect of DSS on mucosal epithelial cells is a key feature of the model. ${ }^{14}{ }^{15}$ Rats were administered $4 \%$ (wt/vol) DSS (molecular weight 40000 daltons; ICN Biomedicals, Aurora, Ohio, USA) in tap water ad libitum for five days. On day 5, rats were killed by cervical dislocation, and the entire colon was dissected, opened longitudinally, fixed in formalin, and coded for blind microscopic assessment of mucosal lesions. Specimens were embedded in paraffin wax and longitudinal sections from the entire specimen (from caecum to rectum) were obtained and 
Table 1 Criteria for microscopic assessment of colonic damage

\begin{tabular}{ll}
\hline Severity of crypt destruction & Grade of injury \\
\hline${ }^{\mathrm{a} C r y p t ~ l e s i o n ~}$ & \\
Intact crypt & 0 \\
Loss of basal third & 2 \\
Loss of two thirds & 3 \\
Loss of entire crypt & 4 \\
Erosion & \\
${ }^{\mathrm{b}}$ Damaged area & 1 \\
$1-25 \%$ & 2 \\
$25-50 \%$ & 3 \\
$50-75 \%$ & 4 \\
$75-100 \%$ & \\
${ }^{\mathrm{C}}$ Inflammation & 0 \\
Absent & 1 \\
Light & 2 \\
Moderate & 3 \\
Severe & \\
${ }^{\mathrm{d}}$ Crypt distortion & 0 \\
Absent & 1 \\
Light & 2 \\
Moderate & 3 \\
Severe & \\
\hline
\end{tabular}

Final score: $(\mathrm{a} \times \mathrm{b})+\mathrm{c}+\mathrm{d}$.

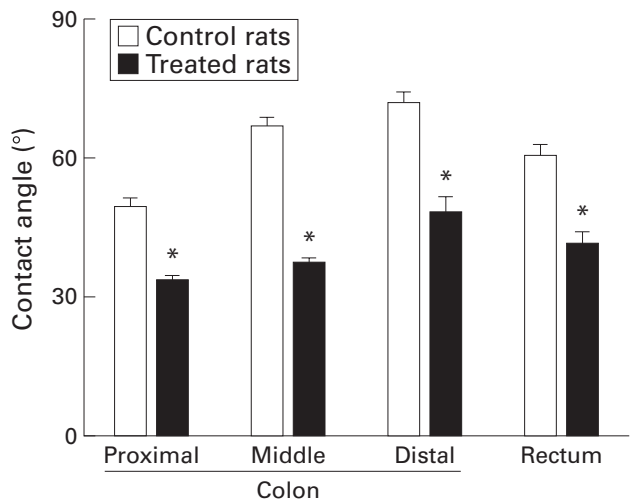

Figure 1 Surface hydrophobicity of the colonic mucosa in control rats and in rats treated with $2 \%$ Brij 35 in the drinking water for two days. Data are presented as the mean (SEM) of five rats per group. ${ }^{*} p<0.05$ compared with control.

stained with haematoxylin and eosin. Two pathologists (AS and JC) examined and scored all sections according to the criteria published by Cooper $e t a l .{ }^{16}$ The score combines severity of crypt destruction with extension of each grade of injury throughout the entire colon (table 1). In ancillary experiments, subsets of five rats were killed on days $0,1,3$, and 5 for measurement of mucosal surface hydrophobicity and colonic permeability as described above.

STATISTICAL METHODS

Results are expressed by the mean and the standard error of the mean. Statistical differences between groups were determined using overall analysis of variance, and the StudentNewman-Keuls method as post test.

\section{Results}

MUCOSAL SURFACE HYDROPHOBICITY

In untreated control rats, surface hydrophobicity was higher at the distal colon than at proximal sites. Contact angles read on top of the caecal mucosa were very low $\left(34(3)^{\circ}, n=30\right)$ in all experiments, and remained unchanged after the treatments tested in this study. Figure 1 shows mucosal surface hydrophobicity in con-

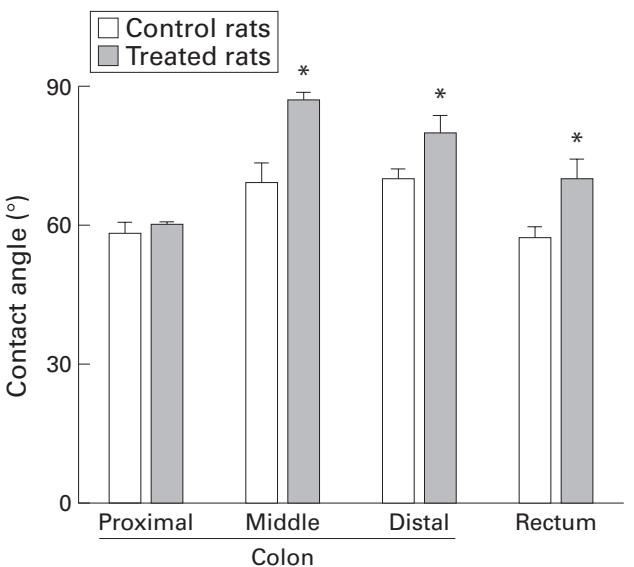

Figure 2 Surface hydrophobicity of the colonic mucosa in control rats and in rats treated with $2 \mathrm{ml}$ of tripalmitin-phosphatidylcholine. Data are presented as the mean (SEM) of seven rats per group. ${ }^{\star} p<0.05$ compared with control.

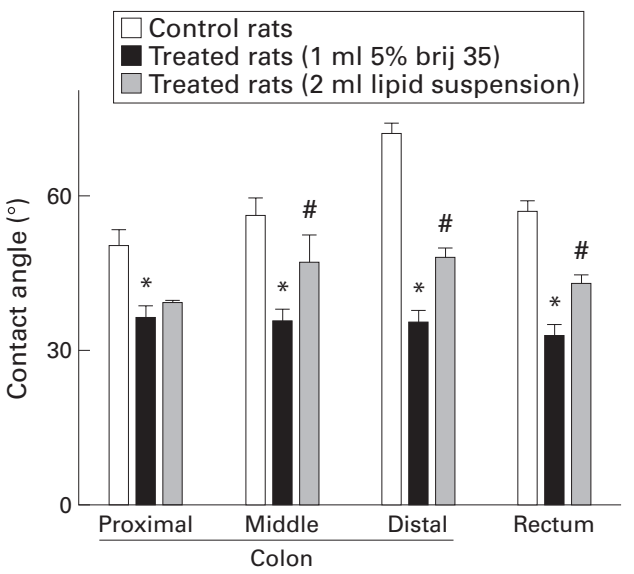

Figure 3 Surface hydrophobicity of the colonic mucosa in control rats, in rats treated with $1 \mathrm{ml} 5 \%$ Brij 35 instilled into the colonic lumen, and in rats treated with 5\% Brij 35 as above and $2 \mathrm{ml}$ of a lipid suspension

(tripalmitin-phosphatidylcholine) 20 minutes later. Data are presented as the mean (SEM) of five rats per group. ${ }^{\star} p<0.05$ compared with control; $\# p<0.05$ compared with Brij 35 only.

trol rats and in rats treated with the detergent Brij 35. The figure shows an important and significant drop in surface hydrophobicity from proximal colon to rectum in rats treated orally with Brij 35. Likewise, $2 \mathrm{ml}$ enemas of $5 \%$ Brij 35 induced a significant reduction of colonic surface hydrophobicity compared with saline enemas (data not shown). Colonic surface hydrophobicity returned to normal contact angles one day after discontinuation of treatment with the detergent.

The effect of topical lipids on colonic surface hydrophobicity was tested in anaesthetised rats. The colon was cannulated at the hepatic flexure and the luminal contents were removed by injection of Krebs-Ringer-Tris solution and air. Thereafter, $2 \mathrm{ml}$ of a suspension of tripalmitin-phosphatidylcholine were instilled in test rats, and $2 \mathrm{ml}$ of saline vehicle in controls. Mucosal surface hydrophobicity was measured 60 minutes after treatment. As fig 2 shows, the lipid suspension induced a notable increase in surface hydrophobicity from midcolon to rectum. Additional experiments tested 

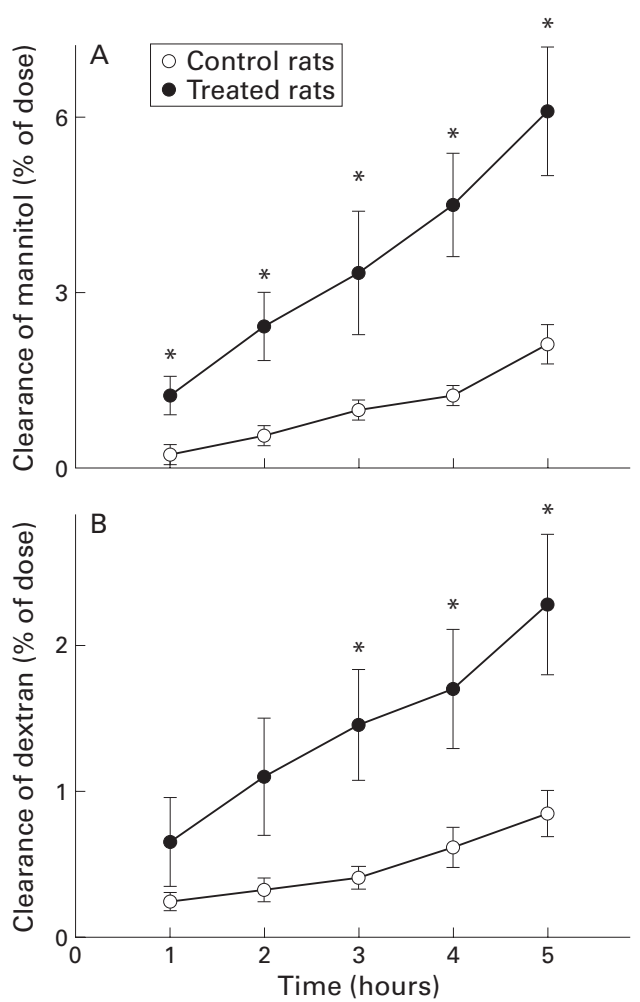

Figure 4 Lumen to blood clearance of mannitol or dextran expressed as percentage of the dose administered

intracolonically at time 0 to control rats and to rats treated with $2 \%$ Brij 35 in the drinking water for two days. Values are means (SEM) for five rats per group. ${ }^{*} p<0.05$ compared with control.

the effect of the lipid suspension in rats pretreated with the detergent ( $5 \%$ Brij 35 , as a bolus through the colonic cannula). As fig 3 shows, the lipid suspension partly reverted the effect of Brij 35 and restored the hydrophobic status of the colonic mucosal surface.

COLONIC PERMEABILITY

Figure 4 shows lumen to blood clearance of mannitol (low molecular size probe) and dextran (high molecular size probe) in control rats and in rats pretreated with Brij 35. Accumulation of the probes mainly reflects the rate of lumen to blood clearance of the molecules, since rats had been nephrectomised previously by ligation of both renal pedicles and the compounds are not metabolised. Clearance of both mannitol and dextran after the five hour study period was notably and significantly increased in Brij 35 rats compared with controls. This finding suggests an enhanced epithelial permeability after treatment with the detergent.

Figure 5 shows the effect of the lipid suspension on colonic permeability. As above, the lipid suspension was administered to anaesthetised rats as a $2 \mathrm{ml}$ bolus through a cannula placed at the hepatic flexure, after removing colonic contents by instillation of KrebsRinger-Tris solution and air. Control rats received $2 \mathrm{ml}$ saline vehicle. No difference was found between the groups in the passage of mannitol from the colonic lumen to circulating blood. However, clearance of dextran was
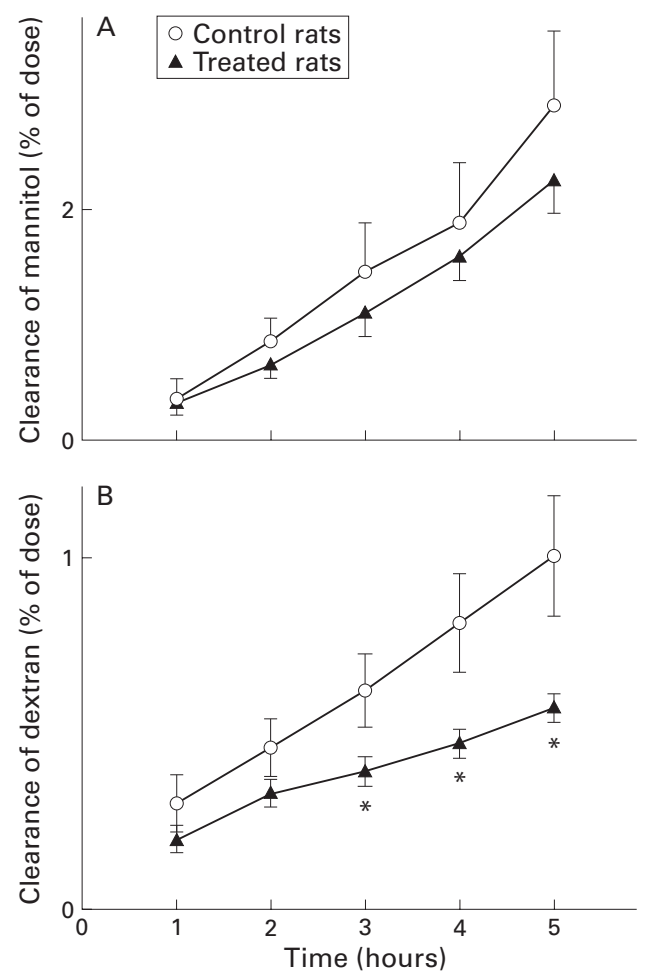

Figure 5 Lumen to blood clearance of mannitol or dextran expressed as percentage of the dose administered

intracolonically at time 0 to control rats and to rats treated with $2 \mathrm{ml}$ of a lipid suspension

(tripalmitin-phosphatidylcholine) instilled into the colonic lumen. Values are means (SEM) for seven rats per group. ${ }^{*} p<0.05$ compared with control.

significantly reduced in rats pretreated with the lipid suspension compared with controls.

MUCOSAL RESISTANCE AGAINST LUMINAL AGGRESSION

Administration of $4 \%$ DSS in the drinking water resulted in a progressive loss of the hydrophobic character of the mucosa in middle and distal colon, as shown in fig 6 . The fall in surface hydrophobicity was associated with increased colonic permeability to the radioactive probes (fig 7). Histological examination of longitudinal colonic sections showed loss of normal crypt architecture, oedema, and diffuse infiltration of inflammatory cells in middle and distal colon on days 3 and 5 after starting DSS in the drinking water. Epithelial erosions were mostly observed at day 5 . However, on days 0 and 1 no significant mucosal lesions were found. Rats treated with DSS for one day showed normal mucosal histology but a deranged surface hydrophobicity and an increased colonic permeability.

To test the role of the surface active phospholipid on mucosal protection against DSS, a group of rats received 2\% Brij 35 in tap water for two days whereas the control group received normal tap water. Thereafter, both groups of rats were treated with $4 \%$ DSS for five days. In parallel experiments, a TP-PC group received daily $2 \mathrm{ml}$ enemas of the lipid suspension for three days before DSS and during the five days of exposure to DSS. The control group received daily $2 \mathrm{ml}$ saline enemas. Figure 8 shows colonic damage scores of the 


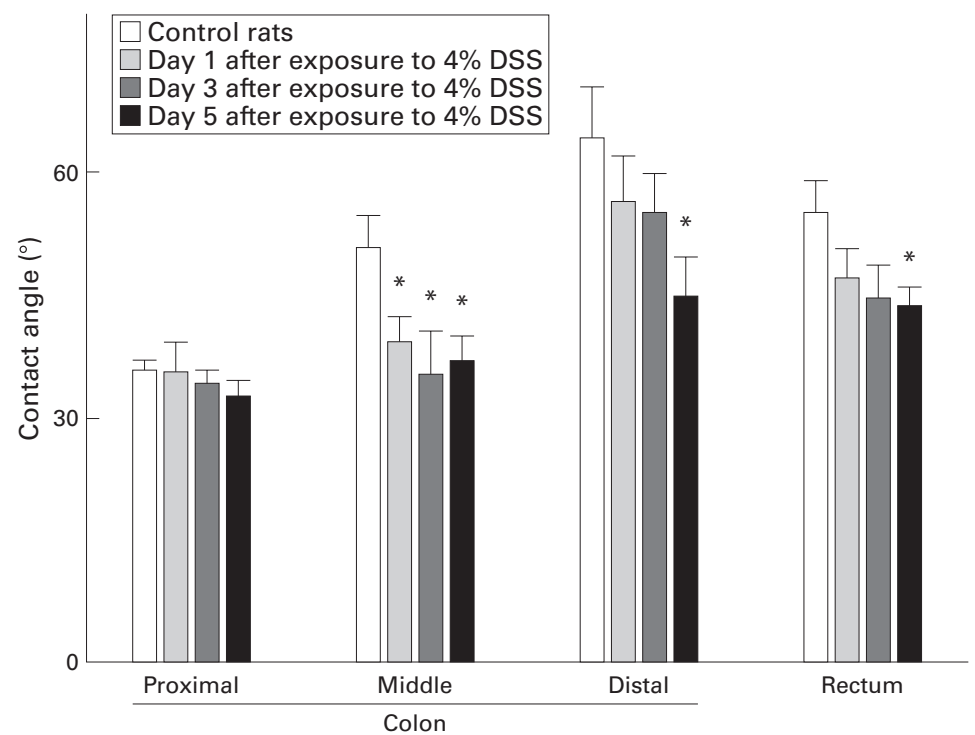

Figure 6 Surface hydrophobicity of the colonic mucosa in control rats (day 0) and in rats treated with $4 \%$ dextran sodium sulphate (DSS) in the drinking water on days 1,3, and 5 after exposure to DSS. Values are means (SEM) for five rats per group. ${ }^{\star} p<0.05$ compared with control.

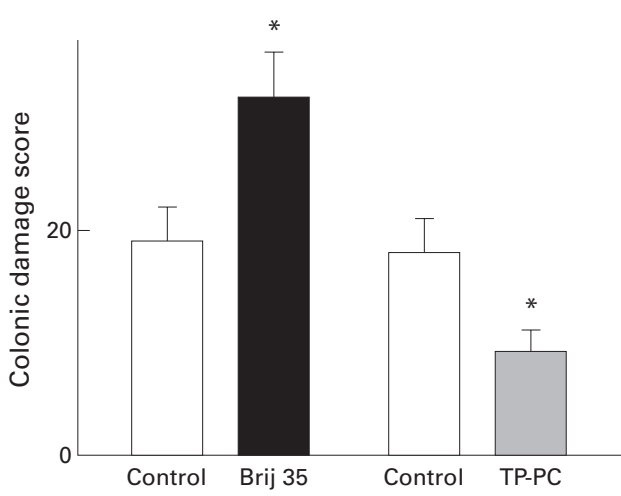

Figure 8 Colonic damage scores after administration of $4 \%$ DSS for five days in control rats, in rats pretreated with $2 \%$ Brij 35 for two days, and in rats treated with $2 \mathrm{ml}$ enemas of a lipid suspension for three days before DSS and during the five days on DSS. Data are presented as the mean (SEM) of five rats per group. ${ }^{\star} p<0.05$ compared with control.

In the rat, contact angles on top of the caecal mucosa were low and similar to those measured on absorptive surfaces such as the duodenal mucosa. ${ }^{12}$ In contrast, specimens from midcolon, left colon, and rectum showed a high surface hydrophobicity. In addition, we found that a non-ionic detergent (Brij 35) can reduce surface hydrophobicity on the colonic mucosa, and conversely, topical administration of a suspension of surface active lipids significantly increases surface hydrophobicity of the distal colon. The effect of the detergent Brij 35 can be reversed by administration of surface active lipids. These observations are consistent with the concept that surface hydrophobicity is mainly owing to a layer of surface active phospholipid which lines the top of the mucus that covers the epithelium, as supported by a number of studies. ${ }^{2-617}$

Our study investigated the functional implication of the surface active phospholipid layer in the mucosal barrier. We observed a derangement of mucosal barrier function in rats treated with the detergent Brij 35. The dysfunction of the intestinal mucosal barrier consisted of an increased permeability to hydrophilic molecules of both small and large size (182 to 70000 daltons), and an increased susceptibility to mucosal inflammation induced by exposure to DSS (40000 daltons). It must be stressed that the detergent Brij 35 by itself at the dose and time of administration tested in this study did not induce any epithelial damage or mucosal inflammation that could be recognised by careful examination at light microscopy.

Parallel experiments showed an enhancement of the mucosal barrier function in rats pretreated with surface active lipids. We prepared a suspension of tripalmitin and dipalmitoyl-phosphatidylcholine that was previously shown to protect the gastric mucosa against direct irritation by a variety of ulcerogenic agents, ${ }^{11}$ and to prevent acid induced mucosal lesions in the proximal duodenum. ${ }^{3}$ In the current study, intracolonic administration of the lipid suspension reduced mucosal permeability to dextran and protected the colonic mucosa against injury by DSS. Thus, 
our experiments suggest that the surface active phospholipid layer plays an important role in the colonic mucosal barrier by providing a physicochemical structure that prevents the penetration of macromolecules into the intestinal wall and the systemic circulation.

The mechanism by which DSS induces mucosal inflammation in the distal colon is not fully understood, but most studies suggest that DSS exhibits a direct toxicity against colonic epithelial cells. Histopathological studies have shown that crypt destruction and loss is the primary event that leads to mucosal inflammatory changes, suggesting that the initial insult is at the level of the epithelial cell. ${ }^{16}$ Direct cytotoxicity of DSS towards intestinal epithelial cells has been shown in vitro. ${ }^{15}$ Acute DSS colitis does not require the presence of $\mathrm{T}$ cells or $\mathrm{B}$ cells because it can be induced in severe combined immunodeficiency mice. ${ }^{18}$ However, chronic colitis after exposure to DSS is characterised by a Th 1 as well as Th 2 cytokine profile. ${ }^{19}$ Immune activation may play a role in the pathogenesis of the chronic phase of DSS induced colitis, but acute colitis by DSS seems to be the result of direct epithelial cell injury. Protection of the mucosal surface against the topical effect of DSS may explain our findings in the experiments using Brij 35 and exogenous lipids.

In our study, oral administration of DSS induced a progressive loss of the hydrophobic character of the distal colonic mucosa, that was accompanied by a progressive derangement of mucosal permeability to mannitol and dextran. We found that changes in hydrophobicity and permeability appeared very early in the course of induction of colitis by DSS and were also observed in rats with normal mucosal histology. It is not known whether DSS may chemically interact with the surface active phospholipid layer as has been shown for the hapten TNBS, ${ }^{10}$ a chemical widely used for induction of experimental colitis. ${ }^{20}$ Alternatively, toxicity of DSS on colonocytes might impair the secretion of surfactant like particles towards the mucosal surface, ${ }^{17}$ before the cell necrosis becomes manifest. However, the interesting finding in our study is that a permeable mucosal barrier is an early event in acute DSS colitis, and that modulation of the strength of the surface active phospholipid layer can modify the course of the experimental disease. Pretreatment with the detergent increased susceptibility to DSS, whereas pretreatment with exogenous surfactant mitigated the onset of colitis.

Our experiments with the DSS model point out that intervention on the surface active phospholipid layer may be a novel therapeutic target for the prevention of inflammatory bowel diseases of the colon. Alterations in intestinal barrier function have been described in a large number of studies of inflammatory bowel diseases. $^{21-24}$ Increased intestinal permeability might not be a primary aetiological factor in inflammatory bowel diseases, but it is certainly an abnormal feature of these disorders that correlates with disease severity. ${ }^{22} 23$ Experimental models of colitis also show an altered permeability to luminal probes. ${ }^{25}$ Indeed, restoration of the mucosal barrier might lead to remission of inflammatory activity, as the uptake of luminal antigens stimulates inflammatory responses within the intestinal wall. ${ }^{27-29}$ For instance, prostaglandins and the epidermal growth factor family of related peptides stimulate the release of surface active phospholipid, ${ }^{3031}$ and they have also been proved effective in mucosal protection and healing of intestinal inflammatory lesions. ${ }^{32-35}$ Reinforcement of surface hydrophobicity might be an effective approach for future strategies in the prevention and treatment of inflammatory bowel diseases.

This research was supported by grant SAF 95-187 from Comision Interministerial para la Investigación Científica y Técnica (Madrid, Spain)

1 Hills BA, Butler BD, Lichtenberger LM. Gastric mucosal barrier: hydrophobic lining to the lumen of the stomach. barrier: hydrophobic lining to the

2 Lichtenberger LM. The hydrophobic barrier properties of gastrointestinal mucus. Annu Rev Physiol 1995;57:565-83.

3 Lugea A, Mourelle M, Guarner F, et al. Phosphatidylcholines as mediators of adaptive cytoprotection of the rat duodenum. Gastroenterology 1994;107:720-7.

4 Butler BD, Lichtenberger LM, Hills BA. Distribution of surfactants in the canine gastrointestinal tract and their ability to lubricate. Am f Physiol 1983;244:G645-51.

5 Goddard PJ, Kao YJ, Lichtenberger LM. Luminal surface hydrophobicity of canine gastric mucosa is dependent on a
surface mucous gel. Gastroenterology 1990;98:361-70.

6 Kao YJ, Goddard PJ, Lichtenberger LM. Morphological effects of aspirin and prostaglandin on the canine gastric mucosal surface. Gastroenterology 1990;98:592-606.

7 Hills BA, Kirwood CA. Gastric mucosal barrier to hydrogen ions imparted by gastric surfactant in vitro. Gut 1992;33: ions impa $1039-41$.

8 Mack DR, Neumann AW, Policova Z, et al. Surface hydrophobicity of the intestinal tract. Am $f$ Physiol 1992;262:G171-7.

9 Spychal RT, Marrero JM, Saverymuttu SH, et al. Measurement of the surface hydrophobicity of human gastrointestinal mucosa. Gastroenterology 1989;97:104-11.

10 Tatsumi Y, Lichtenberger LM. Molecular association of trinitrobenzene-sulfonic acid and surface phospholipids in the development of colitis in rats. Gastroenterology 1996; 110:780-9.

11 Lichtenberger LM, Romero JJ, Kao YJ, et al. Gastric protective activity of mixtures of saturated polar and neutral lipids in rats. Gastroenterology 1990;99:311-26.

12 Lugea A, Antolín M, Mourelle M, et al. Deranged hydrophobic barrier of the rat gastroduodenal mucosa after parenteral nonsteroidal antiinflammatory drugs. Gastroenterology 1997;112:1931-9.

13 Goddard PJ, Hills BA, Lichtenberger LM. Does aspirin damage canine gastric mucosa by reducing its surface damage canine gastric mucosa by reducing its
hydrophobicity? Am f Physiol 1987;15:G421-30.

14 Okayasu I, Hatakeyama S, Yamada M, et al. A novel method in the induction of reliable experimental acute and chronic ulcerative colitis in mice. Gastroenterology 1990;98:694702 .

15 Ni J, Chen SF, Hollander D. Effects of dextran sulphate sodium on intestinal epithelial cells and intestinal lymphocytes. Gut 1996;39:234-41

16 Cooper HS, Murthy SN, Shah RS, et al. Clinicopathologic study of dextran sulfate sodium experimental murine colitis. Lab Invest 1993;69:238-49.

17 Eliakim R, Goetz GS, Rubio S, et al. Isolation and characterization of surfactant-like particles in rat and human colon. Am f Physiol 1997;272:G425-34.

18 Dieleman LA, Ridwan BU, Tennyson GS, et al. Dextran sulfate sodium-induced colitis occurs in severe combined immunodeficient mice. Gastroenterology 1994;107:164352 .

19 Dieleman LA, Palmen MJ, Akol H, et al. Chronic experimental colitis induced by dextran sulphate sodium (DSS) is characterized by Th1 and Th2 cytokines. Clin Exp Immunol 1998;114:385-91.

20 Morris GP, Beck PL, Herridge MS, et al. Hapten-induced model of chronic inflammation and ulceration in the rat colon. Gastroenterology 1989;96:795-803

21 Hollander D, Vadheim CM, Brettholz E, et al. Increased intestinal permeability in patients with Crohn's disease and their relatives. Ann Intern Med 1986;105:883-5.

22 Casellas F, Aguadé S, Soriano B, et al. Intestinal permeability to $99 \mathrm{mTc}$ DPTA in inflammatory bowel disease. Am $\mathcal{F}$ Gastroenterol 1986;81:767-70.

23 Jenkins RT, Jones DB, Goodacre RL, et al. Reversibility of increased intestinal permeability to ${ }^{51} \mathrm{Cr}$-EDTA in patients with gastrointestinal inflammatory disease. Am f Gastroenwerol 1987;82:1159-64.

24 Teahon K, Smethurst P, Levi AJ, et al. Intestinal permeability in patients with Crohn's disease and their first degree relatives. Gut 1992;33:320-3. 
25 Gardiner KR, Anderson NH, Rowlands BJ, et al. Colitis and colonic mucosal barrier dysfunction. Gut 1995;37:530-5.

26 Stein J, Ries J, Barrett KE. Disruption of intestinal barrier function associated with experimental colitis: possible role of mast cells. Am f Physiol 1998;274:G203-9.

27 Jung HC, Eckmann L, Yang SK, et al. A distinct array of proinflammatory cytokines is expressed in human colon epithelial cells in response to bacterial invasion. F Clin Invest 1995;95:55-65.

28 Zareie M, McKay DM, Kovarik GG, et al. Monocyte/ macrophages evoke epithelial dysfunction: indirect role of tumor necrosis factor-alpha. Am f Physiol 1998;275:C9329.

29 Videla S, Vilaseca J, Guarner F, et al. Stimulation of mucosal inflammatory activity by the normal fecal flora in a rat model of colitis. Inflamm Bowel Dis 1997;3:191-7.

30 Scheiman JM, Kraus ER, Bonnville LA, et al. Synthesis and prostaglandin $\mathrm{E}_{2}$-induced secretion of surfactant phospholipid by isolated gastric mucous cells. Gastroenterology 1991;100:1232-40.
31 Lugea A, Guarner F, Malagelada JR, et al. Surface hydrophobicity of the human colonic mucosa: role of epidermal growth factor [abstract]. Gastroenterology 1998; 114:A892.

32 Tessner TG, Cohn SM, Schloemann S, Stenson WF. Prostaglandins prevent decreased epithelial cell proliferation associated with dextran sodium sulfate injury in mice. Gastroenterology 1998;115:874-82.

33 Procaccino F, Reisnhagen M, Hoffmann P, et al. Protective effect of epidermal growth factor in an experimental model effect of epidermal growth factor in an experiment
of colitis in rats. Gastroenterology 1994;107:12-17.

34 Egger B, Procaccino F, Lakshmanan J, et al. Mice lacking transforming growth factor alpha have an increased susceptibility to dextran sulfate-induced colitis. Gastroenterology 1997;113:825-32.

35 Egger B, Carey HV, Procaccino F, et al. Reduced susceptibility of mice overexpressing transforming growth factor alpha to dextran sodium sulphate induced colitis. Gut 1998;43:64-70. 